\title{
Graphene Nanobubbles as Valley Filters and Beam Splitters
}

\author{
Settnes, Mikkel; Power, Stephen; Brandbyge, Mads; Jauho, Antti-Pekka
}

Published in:

Physical Review Letters

Link to article, DOI:

10.1103/PhysRevLett.117.276801

Publication date:

2016

Document Version

Publisher's PDF, also known as Version of record

Link back to DTU Orbit

Citation (APA):

Settnes, M., Power, S., Brandbyge, M., \& Jauho, A-P. (2016). Graphene Nanobubbles as Valley Filters and Beam Splitters. Physical Review Letters, 117(27), [276801]. https://doi.org/10.1103/PhysRevLett.117.276801

\section{General rights}

Copyright and moral rights for the publications made accessible in the public portal are retained by the authors and/or other copyright owners and it is a condition of accessing publications that users recognise and abide by the legal requirements associated with these rights.

- Users may download and print one copy of any publication from the public portal for the purpose of private study or research.

- You may not further distribute the material or use it for any profit-making activity or commercial gain

- You may freely distribute the URL identifying the publication in the public portal

If you believe that this document breaches copyright please contact us providing details, and we will remove access to the work immediately and investigate your claim. 


\title{
Graphene Nanobubbles as Valley Filters and Beam Splitters
}

\author{
Mikkel Settnes, ${ }^{1,2, *}$ Stephen R. Power, ${ }^{1,3}$ Mads Brandbyge, ${ }^{1}$ and Antti-Pekka Jauho ${ }^{1}$ \\ ${ }^{1}$ Center for Nanostructured Graphene $(C N G)$, Department of Micro- and Nanotechnology Engineering, \\ Technical University of Denmark, DK-2800 Kgs. Lyngby, Denmark \\ ${ }^{2}$ Department of Photonics Engineering, Technical University of Denmark, DK-2800 Kgs. Lyngby, Denmark \\ ${ }^{3}$ Department of Physics and Nanotechnology, Aalborg University, DK-9220 Aalborg, Denmark
}

(Received 11 August 2016; published 28 December 2016)

\begin{abstract}
The energy band structure of graphene has two inequivalent valleys at the $K$ and $K^{\prime}$ points of the Brillouin zone. The possibility to manipulate this valley degree of freedom defines the field of valleytronics, the valley analogue of spintronics. A key requirement for valleytronic devices is the ability to break the valley degeneracy by filtering and spatially splitting valleys to generate valley polarized currents. Here, we suggest a way to obtain valley polarization using strain-induced inhomogeneous pseudomagnetic fields (PMFs) that act oppositely on the two valleys. Notably, the suggested method does not involve external magnetic fields, or magnetic materials, unlike previous proposals. In our proposal the strain is due to experimentally feasible nanobubbles, whose associated PMFs lead to different real space trajectories for $K$ and $K^{\prime}$ electrons, thus allowing the two valleys to be addressed individually. In this way, graphene nanobubbles can be exploited in both valley filtering and valley splitting devices, and our simulations reveal that a number of different functionalities are possible depending on the deformation field.
\end{abstract}

DOI: 10.1103/PhysRevLett.117.276801

A remarkable feature of Dirac fermions in graphene is the unique coupling between mechanical deformation and electronic structure. Deforming the graphene lattice introduces an effective gauge field $\boldsymbol{A}$ in the low energy Dirac spectrum [1,2], causing a pronounced sublattice polarization [3-6]. One can associate a pseudomagnetic field (PMF) with this gauge field, $\boldsymbol{B}_{s}=\boldsymbol{\nabla} \times \boldsymbol{A}$. The presence of constant PMFs in graphene has been spectacularly illustrated by scanning tunneling experiments, revealing signatures of Landau quantization [7-10]. In contrast to the usual constant PMFs, in this Letter we focus on spatially varying PMFs, and show that inhomogenous PMFs can be used as a building block for valleytronic devices.

Unlike real magnetic fields, strain-induced PMFs conserve time-reversal symmetry and take opposite signs in the $K$ and $K^{\prime}$ valleys [11,12]. The effective gauge field enters the low energy Dirac Hamiltonian $H=v_{F} \boldsymbol{\sigma} \cdot \boldsymbol{p}$ via the transformation $\boldsymbol{p} \rightarrow \boldsymbol{p} \pm \boldsymbol{e A}$, where \pm denote either the $K$ or $K^{\prime}$ valley [11,12]. This sign difference between $K$ and $K^{\prime}$, together with the spatially varying PMF, lies at the heart of our suggestion to manipulate the valley degree of freedom using strain engineering.

Different routes have been suggested to create valley polarization in graphene [13-17], relying on nanoribbons or constrictions [17-21], interplays between external fields [22-25], spin-orbit coupling [26,27], or spatial or temporal combinations of gating and magnetic fields [28-30]. However, an experimental verification has proven to be challenging as practical and effective methods to manipulate the valleys in realistic setups still need to be established.
In this Letter, we show that experimentally feasible local strain fields due to local deformations give rise to PMFs that allow for valley control without the need for additional fields or interactions. Experimental methods for producing such controllable strain fields include direct applied pressure from STM tips [31], gas inflation [32-36], and substrate engineering [37-45]. Most of these approaches result in spatially localized strain fields taking the form of a pseudomagnetic dot. The PMFs created this way are usually of great magnitude and local but spatially varying. We show that such systems can exhibit strong valley dependent effects associated with two key valleytronic components-namely, valley filters (as illustrated in Fig. 1) and beam splitters, which spatially separate the different valleys (see Fig. 4).

Methodology.-The electronic structure of strained graphene is treated using a first nearest neighbor tight binding model $\boldsymbol{H}=-\sum_{\langle i, j\rangle} t_{i j} c_{i}^{\dagger} c_{j}$, where the sum $\langle i, j\rangle$ runs over nearest neighbors. Strain is included by modifying the hopping parameter such that $t_{i j}=t_{0} \exp \left[-\beta\left(d_{i j} / a_{0}-1\right)\right]$ [46], where $a_{0}=0.142 \mathrm{~nm}, \beta=3.37, t_{0}=-2.7 \mathrm{eV}$, and $d_{i j}$ is the modified bond length. In this way, we do not use the Dirac model in the actual calculations but only to interpret the results from the full tight binding calculation.

We apply the patched Green's function approach $[47,48]$ to calculate the response of a plane electron wave impinging on the strained nanobubble. Using the patched Green's function method, we replace the infinite graphene Hamiltonian by a finite effective Hamiltonian $\boldsymbol{H}_{\text {eff }}=\boldsymbol{H}+\Sigma_{B}$, where $\boldsymbol{H}$ describes a finite patch of the system and the self-energy 


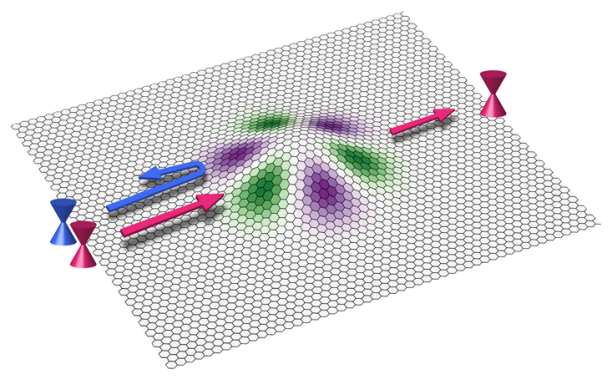

FIG. 1. An incoming incoming electron wave containing both $K$ and $K^{\prime}$ valleys incident on a Gaussian nanobubble experiences the associated pseudomagnetic field indicated by the (green and purple) color map. $K$ valley electrons are backscattered whereas those from the $K^{\prime}$ valley are transmitted due to the different trajectories imposed by the effective magnetic field for each valley when electrons are incident along a specific direction relative to the field.

$\Sigma_{B}$ contains the influence of the surrounding infinite, pristine graphene sheet upon the patch. The full Green's function for the patch region becomes $\boldsymbol{G}(E)=\left(E-\boldsymbol{H}-\Sigma_{B}-\Sigma_{L}\right)^{-1}$, where $\Sigma_{L}$ is the lead self-energy describing a pointlike metallic probe with a constant density of state emitting an electron wave with a mixture of both valleys [47]. The probe is placed $250 \mathrm{~nm}$ away from the deformations such that the impinging wave approximately becomes a plane wave.

$\Sigma_{B}$ is expressed conveniently using pristine Green's functions along the boundary of the calculation area exploiting complex contour techniques $[48,49]$. To calculate the Green's function and the local current $J_{i j}=\operatorname{Im}\left(t_{i j} A_{i j}\right) / \hbar$, we employ an adaptive recursive routine [47]. Here, the spectral function $A_{i j}$ is defined as $A_{i j}=\left(\boldsymbol{G} \boldsymbol{\Gamma}_{L} \boldsymbol{G}^{\dagger}\right)_{i j}$, where the broadening due to the lead is $\Gamma_{L}=i\left(\Sigma_{L}-\Sigma_{L}^{\dagger}\right)$.

To determine the valley occupation of the electron wave, we consider the outgoing scattering state in real space given by the spectral function $A_{i j}$ [50]. We expand this scattering state in the basis of the pristine eigenstate of graphene $|\boldsymbol{k}, \lambda\rangle$ ( $\lambda$ is the band index) [51] In this way, the matrix element $c_{\boldsymbol{k}}=\langle\boldsymbol{k}, \lambda|\boldsymbol{A}| \boldsymbol{k}, \lambda\rangle$ becomes a spectral density in $\boldsymbol{k}$ space indicating the $\boldsymbol{k}$ values occupied by the real space scattering state. The element $c_{k}$ can then be computed for each $\boldsymbol{k}$ value separately to produce a Fourier map of the scattering state illustrating the full valley occupation.

Gaussian deformation for valley polarization.-We first consider a Gaussian deformation [2,52,53] corresponding to an out-of-plane displacement $z(r)=h_{0} \exp \left(-r^{2} / 2 \sigma^{2}\right)$, where $h_{0}=3.5 \mathrm{~nm}$ and $\sigma=5 \mathrm{~nm}$ are the height and width of the deformation, corresponding to a maximum strain of approximately $8.5 \%$. The results are robust and scalable for other deformation dimensions. This circularly symmetric deformation gives rise to a PMF distribution indicated by the color map in Fig. 2(a) for the $K$ valley; an equally strong field but of opposite sign is experienced by the $K^{\prime}$ valley. The classical circular trajectories, forming a vortex pattern, expected for such a field profile are shown for the $K$ (blue arrows) and $K^{\prime}$ valleys (red arrows). The plane wave is incident along the zigzag direction and the resulting local currents at an energy corresponding to the lowest resonance energy of the Gaussian deformation are shown in Fig. 2(b). A detailed description of the resonances is given in the Supplemental Material [54]. The size and direction of the arrows in Fig. 2(b) indicate the magnitude and direction of the local current. We especially note that the local current is largest at the interface between PMF regions of different sign, suggesting that snake states are formed here in a manner similar to systems with real magnetic fields [55]. From Figs. 2(b) and 2(c) it is also clear that the deformation enhances the current in the region directly behind it, acting as a lens that focuses the current at this electron energy [56].

Comparing the direction of the vortex patterns in Fig. 2(a) and the local current in Fig. 2(b), we find that only the current direction associated with the $K^{\prime}$ valley is visible. This does not, however, imply that the bubble is in a valley-polarized eigenstate. Instead, only one of the trajectories matches the direction of the incoming wave. Thus, we find a pattern matching the $K$ valley vortices for a current incident from the right, or a mixture of both patterns for incidence from the top or bottom (see the Supplemental Material for details [54]). Electrons in the $K$ valley "see" only the vortex pattern indicated by blue arrows in Fig. 2(a), which tends to backscatter electrons incident from the left, and transmit through the dot if incident from the right. Conversely, $K^{\prime}$ electrons see the pattern shown by red arrows, and if incident from the left they are guided through the strained region along snake states between regions with PMFs of opposite sign. Thus, the valley selection mechanism relies on the symmetry breaking caused by the direction of the incoming current and
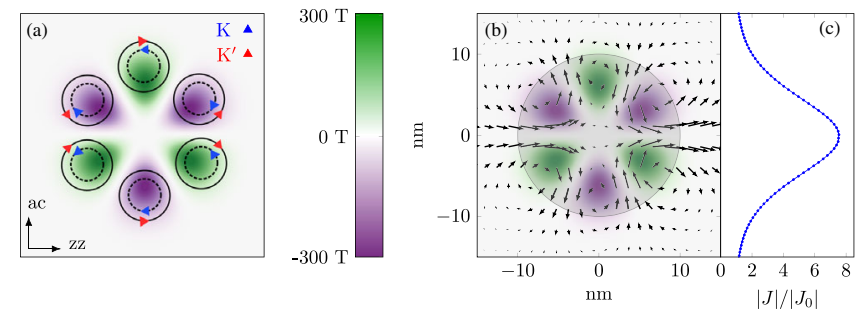

FIG. 2. (a) The color map indicates the threefold symmetric pseudomagnetic field caused by the circularly symmetric Gaussian deformation. The vortices show trajectories corresponding to the field experienced in the $K$ (blue) and $K^{\prime}$ valleys (red). (b) Calculation of the local current incident from the left along the zigzag direction at $E=0.01\left|t_{0}\right|$. The arrows indicate the direction and magnitude of the current. The arrows are averaged over several sites to enhance visibility. The shaded area indicates the $r<2 \sigma$ region. (c), Spatially resolved current density with strain $(|J|)$, relative to that without strain $\left(\left|J_{0}\right|\right)$, evaluated at the rightmost edge of panel (b), demonstrating that the strained region focuses the initially uniform current. 

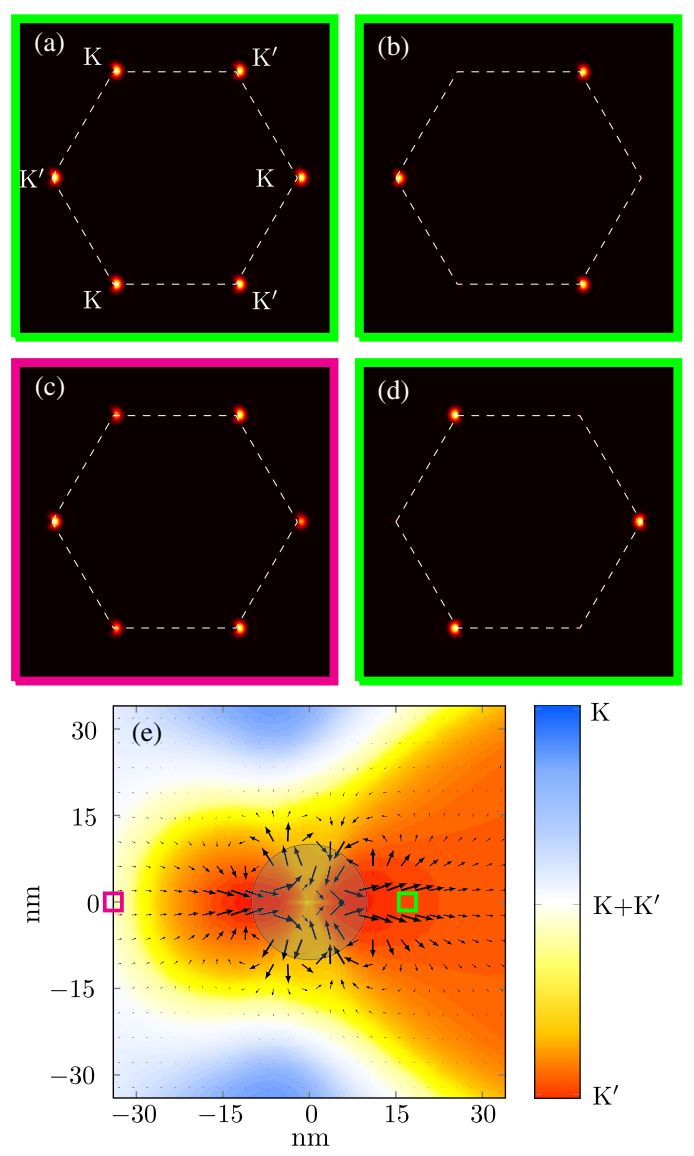

FIG. 3. Panels (a) and (b) compare the $k$-space occupation for $E=0.01\left|t_{0}\right|$ at the green square in panel (e) without (a) and with (b) the presence of the deformation. (c) $k$-space occupation for $E=0.01\left|t_{0}\right|$ at the red square in panel (e). (d) $k$-space occupation at the green square but with negative energy $E=-0.01\left|t_{0}\right|$, showing the reversal of the $k$-filtering effect. (e), Real space map of the relative occupation of $K$ and $K^{\prime}$ in the scattering state showing the real space filtering of the valleys. The local current map from Fig. 2(b) is reproduced for convenience.

not on a valley polarization of the states in the bubble. The presence of states from the opposite valley of course makes the effects discussed here vulnerable to intervalley scattering, such as that induced by short-ranged disorder.

To further examine the valley dependence of the computed current patterns, we calculate the spectral density $\left|c_{k}\right|$ for each $\boldsymbol{k}$ value. Figures 3(a) and 3(b) show such Fourier maps generated for the region indicated by the green box in Fig. 3(e) without [Fig. 3(a)] and with [Fig. 3(b)] the presence of the strain field. The valley filtering occurs when passing through the Gaussian deformation and the transmitted wave consists almost exclusively of electrons in the $K^{\prime}$ valley. On the other hand, Fig. 3(c) shows that both valleys are present (but not with equal weights) in the region before the bubble, shown by the red box. Finally, the full map of the valley occupation [Fig. 3(e)] confirms the earlier intuitive analysis based on the local current trajectories in Fig. 2(a): the $K^{\prime}$ valley totally dominates the strained region while the $K$ valley entirely avoids the strained region.

The valley filtering effect arises due to the different signs of the PMFs experienced by the two valleys. Similarly, the current paths for $E$ and $-E$ are equal, but the opposite energy sign swaps the valleys and the other valley is transmitted or backscattered [as illustrated in Fig. 3(d)]. This intriguing observation opens the desirable possibility of valley selectivity by a simple back gate as the Fermi energy is shifted between positive and negative values. Even further tunability is possible when observing that the valley filtering effect is strongest at energies corresponding to resonances of the deformation. Thus, small adjustments in the Fermi energy allow one to turn on and off the valley filtering effect. In the Supplemental Material [54], we examine how varying a gate allows one to move in and out of resonance with the eigenstates, which are strongly affected by the PMF.

Triaxial deformation for valley splitting.-Finally, we consider an alternative geometry consisting of an in-plane triaxial strain $[1,2,7]$ and additional out-of-plane deformation, appropriate for small bubbles formed by graphene on a substrate [7]. The displacements are

$$
\left(\begin{array}{c}
u_{r} \\
u_{\theta} \\
z
\end{array}\right)=\left(\begin{array}{c}
u_{0} r^{2} \sin (3 \theta) \\
u_{0} r^{2} \cos (3 \theta) \\
h_{0}
\end{array}\right) e^{-\frac{r^{2}}{2 \sigma^{2}}},
$$

where $(r, \theta)$ are polar coordinates $(\theta=0$ corresponding to the zigzag direction). $h_{0}=1 \mathrm{~nm}$ and $\sigma=5 \mathrm{~nm}$ are the height and width of the deformation and $u_{0}$ is the in-plane strength, which is chosen to give a PMF of approximately $300 \mathrm{~T}$ at the center of the deformation. For the chosen deformation size this yields a strain of approximately $2.5 \%$. The resulting PMF distribution is shown in Fig. 4(a) together with effective trajectories for the $K$ (blue arrow) and $K^{\prime}$ valley (red arrow) giving rise to a splitting of the current. The real space valley polarization of the scattering
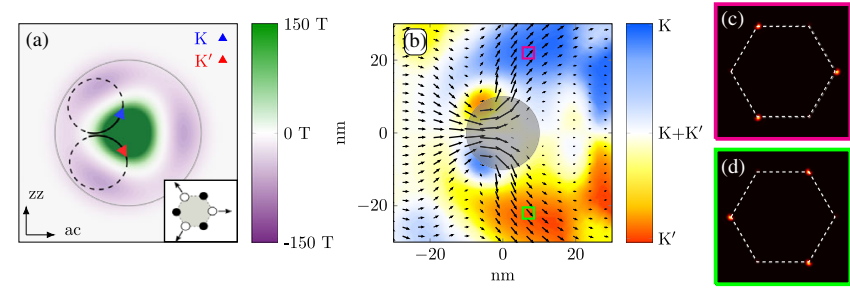

FIG. 4. (a) Pseudomagnetic field distribution for the triaxial displacement field with schematic trajectories shown for the $K$ (blue) and $K^{\prime}$ valley (red). The inset shows the direction of the triaxial strain. (b) Real space map at $E=0.019\left|t_{0}\right|$ of both the local current (arrows) and relative $k$ occupation (color map) of the scattering state incoming from the armchair direction (note the rotation compared to Fig. 3). (c),(d) Fourier maps for the scattering state at the red and green boxes indicated in panel (b). 
state and the local currents are mapped in Fig. 4(b) for one resonant mode of the deformation together with the Fourier maps illustrating the valley splitting in Figs. 4(c) and 4(d). The valley-dependent electron trajectories are again governed by interfaces between regions with different PMF polarity, which support propagation in opposite directions for the two valleys. The net effect of such trajectories is a symmetric splitting of the valleys perpendicular to the incident (armchair) direction. Details of incidence in low symmetry directions, higher order resonance modes, and their resulting trajectories are given in the Supplemental Material [54].

Discussion and conclusion.-Direct experimental confirmation of the valley splitting in an experimental setting has previously been envisioned by employing real magnetic fields to alter the magnitudes, and not just the sign, of the total field experienced by each valley $[23,28]$. The results presented in this Letter, however, open a different route to experimental confirmation. Using individually gated nanobubbles, we can exploit the interchanged roles of the valleys for opposite electron energies. In this way, oppositely gated nanobubbles will filter opposite valleys and effectively block the current while also creating the opportunity to turn on and off the valley polarized current. Furthermore, the valley polarized currents generated by our setup will change the expected degeneracies of currentcarrying states in, for example, Hall effect measurements.

To conclude, we have demonstrated how interfaces between pseudomagnetic fields of different polarity enable valley-dependent guiding of electrons in graphene. The two valleys experience a different field giving rise to different electron trajectories for each valley. The two different strain profiles considered provide illustrative examples of valleyfiltering and valley-splitting devices, allowing for the construction of various valleytronic devices. This suggests alternative routes to experimental observation of valley polarization in graphene as well as a basis for topological valley (Hall) currents, along the lines of recent demonstrations by alternative mechanisms in graphene [57], bilayer graphene [58], and other two-dimensional materials [59-61].

The authors thank N. A. Mortensen and T. Gunst for helpful comments and discussions. The work of M.S is supported by the Danish Council for Independent Research (Grant No. DFF-5051-00011). The Center for Nanostructured Graphene (CNG) is sponsored by the Danish National Research Foundation (Grant No. DNRF103).

*mikse@nanotech.dtu.dk

[1] F. Guinea, M. I. Katsnelson, and A. K. Geim, Nat. Phys. 6, 30 (2010).

[2] F. D. Juan, A. Cortijo, M. A. H. Vozmediano, and A. Cano, Nat. Phys. 7, 810 (2011).
[3] M. Neek-Amal, L. Covaci, K. Shakouri, and F. M. Peeters, Phys. Rev. B 88, 115428 (2013).

[4] M. Schneider, D. Faria, S. Viola Kusminskiy, and N. Sandler, Phys. Rev. B 91, 161407 (2015).

[5] M. Settnes, S. R. Power, and A.-P. Jauho, Phys. Rev. B 93, 035456 (2016).

[6] A. Georgi, P. Nemes-Incze, R. Carrillo-Bastos, D. Faria, S. V. Kusminskiy, D. Zhai, M. Schneider, D. Subramaniam, T. Mashoff, N. M. Freitag, M. Liebmann, M. Pratzer, L. Wirtz, C. R. Woods, R. V. Gorbachev, Y. Cao, K. S. Novoselov, N. Sandler, and M. Morgenstern, arXiv:1611.06123

[7] N. Levy, S. Burke, K. L. Meaker, M. Panlasigui, A. Zettl, F. Guinea, A. Neto, and M. Crommie, Science 329, 544 (2010).

[8] J. Lu, A. H. Castro Neto, and K. P. Loh, Nat. Commun. 3, 823 (2012).

[9] S.-Y. Li, K.-K. Bai, L.-J. Yin, J.-B. Qiao, W.-X. Wang, and L. He, Phys. Rev. B 92, 245302 (2015).

[10] H. Lim, J. Jung, R. S. Ruoff, and Y. Kim, Nat. Commun. 6, 8601 (2015).

[11] M. Vozmediano, M. Katsnelson, and F. Guinea, Phys. Rep. 496, 109 (2010).

[12] V. M. Pereira and A. H. Castro Neto, Phys. Rev. Lett. 103, 046801 (2009).

[13] O. Gunawan, Y. P. Shkolnikov, K. Vakili, T. Gokmen, E. P. De Poortere, and M. Shayegan, Phys. Rev. Lett. 97, 186404 (2006).

[14] O. Gunawan, B. Habib, E. P. De Poortere, and M. Shayegan, Phys. Rev. B 74, 155436 (2006).

[15] D. Xiao, W. Yao, and Q. Niu, Phys. Rev. Lett. 99, 236809 (2007).

[16] D. Gunlycke and C. T. White, Phys. Rev. Lett. 106, 136806 (2011).

[17] A. Rycerz, J. Tworzydlo, and C. W. J. Beenakker, Nat. Phys. 3, 172 (2007).

[18] A. Chaves, L. Covaci, K. Y. Rakhimov, G. A. Farias, and F. M. Peeters, Phys. Rev. B 82, 205430 (2010).

[19] L. S. Cavalcante, A. Chaves, D. R. da Costa, G. A. Farias, and F. M. Peeters, Phys. Rev. B 94, 075432 (2016).

[20] R. Carrillo-Bastos, C. León, D. Faria, A. Latgé, E. Y. Andrei, and N. Sandler, Phys. Rev. B 94, 125422 (2016).

[21] S. P. Milovanović and F. M. Peeters, Appl. Phys. Lett. 109, 203108 (2016)

[22] Z. Qi, D. A. Bahamon, V. M. Pereira, H. S. Park, D. K. Campbell, and A.H. Castro Neto, Nano Lett. 13, 2692 (2013).

[23] T. Low and F. Guinea, Nano Lett. 10, 3551 (2010).

[24] T. Fujita, M. B. A. Jalil, and S. G. Tan, Appl. Phys. Lett. 97, 043508 (2010).

[25] D. R. da Costa, A. Chaves, S. H. R. Sena, G. A. Farias, and F. M. Peeters, Phys. Rev. B 92, 045417 (2015).

[26] M. M. Grujić, M.Z. Tadić, and F. M. Peeters, Phys. Rev. Lett. 113, 046601 (2014).

[27] Q.-P. Wu, Z.-F. Liu, A.-X. Chen, X.-B. Xiao, and Z.-M. Liu, Sci. Rep. 6, 21590 (2016).

[28] Z. Wu, F. Zhai, F. M. Peeters, H. Q. Xu, and K. Chang, Phys. Rev. Lett. 106, 176802 (2011).

[29] Y. Jiang, T. Low, K. Chang, M. I. Katsnelson, and F. Guinea, Phys. Rev. Lett. 110, 046601 (2013). 
[30] D. A. Cosma, M. Mucha-Kruczyński, H. Schomerus, and V. I. Fal'ko, Phys. Rev. B 90, 245409 (2014).

[31] N. N. Klimov, S. Jung, S. Zhu, T. Li, C. A. Wright, S. D. Solares, D. B. Newell, N. B. Zhitenev, and J. A. Stroscio, Science 336, 1557 (2012).

[32] J. S. Bunch, S. S. Verbridge, J. S. Alden, A. M. van der Zande, J. M. Parpia, H. G. Craighead, and P. L. McEuen, Nano Lett. 8, 2458 (2008).

[33] T. Georgiou, L. Britnell, P. Blake, R. V. Gorbachev, A. Gholinia, A. K. Geim, C. Casiraghi, and K. S. Novoselov, Appl. Phys. Lett. 99, 093103 (2011).

[34] Z. Qi, A. L. Kitt, H. S. Park, V. M. Pereira, D. K. Campbell, and A. H. Castro Neto, Phys. Rev. B 90, 125419 (2014).

[35] D. A. Bahamon, Z. Qi, H. S. Park, V. M. Pereira, and D. K. Campbell, Nanoscale 7, 15300 (2015).

[36] J. V. Sloan, A. A. Pacheco Sanjuan, Z. Wang, C. Horvath, and S. Barraza-Lopez, Phys. Rev. B 87, 155436 (2013).

[37] S. Viola Kusminskiy, D. K. Campbell, A. H. Castro Neto, and F. Guinea, Phys. Rev. B 83, 165405 (2011).

[38] A. Reserbat-Plantey, D. Kalita, Z. Han, L. Ferlazzo, S. Autier-Laurent, K. Komatsu, C. Li, R. Weil, A. Ralko, L. Marty, S. Gueron, N. Bendiab, H. Bouchiat, and V. Bouchiat, Nano Lett. 14, 5044 (2014).

[39] G. W. Jones and V. M. Pereira, New J. Phys. 16, 093044 (2014).

[40] M. Neek-Amal and F. M. Peeters, Phys. Rev. B 85, 195445 (2012).

[41] M. Neek-Amal and F. M. Peeters, Phys. Rev. B 85, 195446 (2012).

[42] M. Neek-Amal, L. Covaci, and F. M. Peeters, Phys. Rev. B 86, 041405 (2012).

[43] S. T. Gill, J. H. Hinnefeld, S. Zhu, W. T. Swanson, T. Li, and N. Mason, ACS Nano 9, 5799 (2015).

[44] S. Scharfenberg, D. Z. Rocklin, C. Chialvo, R. L. Weaver, P. M. Goldbart, and N. Mason, Appl. Phys. Lett. 98, eid 091908 (2011).

[45] W. Bao, F. Miao, Z. Chen, H. Zhang, W. Jang, and C. Dames, Nat. Nanotechnol. 4, 562 (2009).

[46] V. M. Pereira, A. H. Castro Neto, and N. M. R. Peres, Phys. Rev. B 80, 045401 (2009).
[47] M. Settnes, S. R. Power, J. Lin, D. H. Petersen, and A.-P. Jauho, Phys. Rev. B 91, 125408 (2015).

[48] S. R. Power and M. S. Ferreira, Phys. Rev. B 83, 155432 (2011).

[49] M. Settnes, S. R. Power, D. H. Petersen, and A.-P. Jauho, Phys. Rev. Lett. 112, 096801 (2014).

[50] M. Paulsson and M. Brandbyge, Phys. Rev. B 76, 115117 (2007).

[51] The pristine eigenstate of the graphene lattice is $|\boldsymbol{k}, \lambda\rangle=\frac{1}{\sqrt{2 N}} \sum_{\boldsymbol{R}_{i}} \mathrm{e}^{-\mathrm{i} \boldsymbol{k} \boldsymbol{R}_{i}}\left[\left|\boldsymbol{R}_{i}, \bullet\right\rangle+\lambda \mathrm{e}^{-\mathrm{i} \phi_{k}}\left|\boldsymbol{R}_{i}, \circ\right\rangle\right]$. where $\boldsymbol{R}_{i}$ is the position of the unit cell, $\lambda= \pm$ is the band index, and $\bullet$ (o) denotes the sublattice $A(B)$. Here, $e^{-i \phi_{k}}=f(\boldsymbol{k})^{*} /|f(\boldsymbol{k})|$ with $f(\boldsymbol{k})=1+\exp \left(i \boldsymbol{k} \boldsymbol{a}_{1}\right)+\exp \left(\boldsymbol{i k \boldsymbol { a } _ { 2 }}\right)$ and the lattice vectors $\boldsymbol{a}_{1}=a_{0}(-\sqrt{3}, 3) / 2$ and $\boldsymbol{a}_{2}=a_{0}(\sqrt{3}, 3) / 2$.

[52] D. Moldovan, M. Ramezani Masir, and F. M. Peeters, Phys. Rev. B 88, 035446 (2013).

[53] R. Carrillo-Bastos, D. Faria, A. Latgé, F. Mireles, and N. Sandler, Phys. Rev. B 90, 041411 (2014).

[54] See Supplemental Material at http://link.aps.org/ supplemental/10.1103/PhysRevLett.117.276801 for details of higher order resonances in the nanobubbles as well as alternative incident directions for the electron wave.

[55] A. De Martino, L. Dell' Anna, and R. Egger, Phys. Rev. Lett. 98, 066802 (2007).

[56] T. Stegmann and N. Szpak, New J. Phys. 18, 053016 (2016).

[57] R. V. Gorbachev, J. C. W. Song, G. L. Yu, A. V. Kretinin, F. Withers, Y. Cao, A. Mishchenko, I. V. Grigorieva, K. S. Novoselov, L. S. Levitov, and A. K. Geim, Science 346, 448 (2014).

[58] Y. Shimazaki, M. Yamamoto, I. V. Borzenets, K. Watanabe, T. Taniguchi, and S. Tarucha, Nat. Phys. 11, 1032 (2015).

[59] Y. D. Lensky, J. C. W. Song, P. Samutpraphoot, and L. S. Levitov, Phys. Rev. Lett. 114, 256601 (2015).

[60] K. F. Mak, K. He, J. Shan, and T. F. Heinz, Nat. Nanotechnol. 7, 494 (2012).

[61] J. Lee, K. F. Mak, and J. Shan, Nat. Nanotechnol. 11, 421 (2016). 